\title{
Table of jurisprudence
}

\section{CANADA}

682330 Ont. Inc. v. Cineplex Odeon

Corp. (1990), 33 C.P.R. (3d) 408

(F.C.T.D.), aff'd (1990), 33

C.P.R. (3d) 413 (F.C.T.D.) 191

AB Hassle v. Apotex Inc., [2006] 4

F.C.R. 513 (F.C.A.), 2006 FCA

$51 \quad \mathbf{7 0}$

ACA Joe International v. 147255

Canada Inc. et al. (1986), 10

C.P.R. (3d) 301 (F.C.T.D.) 9

Anglo-Canadian Music Publisher's

Association v. Dupuis (1903), 27

C.S. 485 (Que. S.C.) 122

Application by Pointe-à-Callière,

Montreal Museum of Archeology

and History for the Reproduction

of Quotations (29 March 2004),

Copyright Board Decision,

online: <http://www.cb-

cda.gc.ca/unlocatable/other/1-

b.pdf $>238$

Armand Vaillancourt v. Carbone 14,

[1999] R.J.Q. 490 (Que. C.S.)

178, 181

Astrazeneca Canada Inc. v. Canada

(Minister of Health), [2006] 2

S.C.R. $560 \quad 67$

Aubry v. Editions Vice-Versa, [1998]

1 S.C.R. $591 \quad$ 247, 252

Banquet \& Catering Supplies Rental

Ltd. v. Bench \& Table Rental

World Inc. (1979), 52 C.P.R. (2d)

71 (Que. S.C.) 9
Bayer Inc. v. Canada (Attorney

General) (1998), 84 C.P.R. (3d)

129, [1999] 1 F.C. 553 (F.C.T.D)

99

Bayliner Marine Corporation v.

Doral Boats Ltd. (1985) 5 C.P.R.

(3d) 289 (F.C.T.D.), rev'd [1986]

3 F.C. 421 (F.C.A.) 271

Beatrice/Hunt-Wesson Inc. v.

Chocosuisse, Union des

Fabricants Suisses de Chocolat

(1992), 45 C.P.R. (3d) 268

(T.M.O.B.) 61

Beatty v. Canada (A.G.) (2004), [2005] 1 F.C.R. 327 [2004] F.C.J. No. $1162 \mathbf{2 4 8}$

Beauchemin v. Cadieux (1900), 10

Que. KB 255, aff'd (1901) 31

S.C.R. $370 \quad \mathbf{1 2 4}$

Belzberg v. British Columbia

Television Broadcasting Ltd

(1981) (unreported, described in

Silber v. British Columbia

Television Broadcasting System

(1985), 25 D.L.R. (4th) 345)

250

Bernard v. Bertoni (1888), 14 Q.L.R.

219 (Que. S.C.) 123

Beullac Ltée. v. Simard (1910), 39

C.S. 97 (Que. S.C.), aff'd (1911)

39 C.S. 517 (Que. S.C.-Revision)

\section{3, 124}

Bingo Enterprises Ltd. v. Plaxton (1986), 26 D.L.R. (4th) 604

(Man. C.A.) 248 
Bishop v. Stevens, [1990] 2 S.C.R. 467238

Black v. The Imperial Book

Company (1904), 8 Ont. L. R. 9 (Ont. C.A.) 113, 136

BMG Canada Inc. v. John Doe, [2005] 4 F.C.R. 81, (2005) 39 C.P.R. (4th) 97, aff'g $B M G$ Canada Inc. v. John Doe, [2004] 3 F.C.R. 241, (2004) 32 C.P.R. (4th) 64 227, 230, 231, 232, 233, 234, 235, 236, 237, 241, 243, 244, 245, 252, 258, 259, 260, 261, 262, 263, 264, 266

Bristol-Myers Squibb Co. v. Canada (Attorney General), [2005] 1 S.C.R. 533, 2005 SCC $26 \quad 67$, 93

British Columbia Automobile Assn.

v. Office and Professional

Employee's International Union (2001), 10 C.P.R. 423 (B.C.S.C.) 32

Cadbury Schweppes Inc. v. FBI

Foods Ltd., [1999] 1 S.C.R. 142 253

Canada Publishing Co. v. Gage (1885), 11 S.C.R. $306 \mathbf{2 8 4}$

Canadian Blood Services/Société du

Sang v. John Doe (17 June 2002),

(Ont. S.C.J.) 261

Canadian Broadcasting Corp. v.

Copyright Appeal Board (1986),

17 C.P.R. (3d) 460 (F.C.A.) 213

Canadian Cable Television

Association v. Canada (Copyright

Board), [1993] 2 F.C. 138

(F.C.A.) 212

Canadian Council of Blue Cross

Plans v. Blue Cross Beauty

Products Inc., [1971] F.C. 543

(F.C.T.D.) 29, 31
Canadian Olympic Association v.

Konica Canada Inc. (1991), 39

C.P.R. (3d) 400 (F.C.A.) 6

Canadian Private Copying

Collective v. Amico Imaging

Services Inc. (2004), 249 F.T.R.

312 (F.C.T.D.) 271

Canadian Private Copying

Collective v. Canadian Storage

Media Alliance (2004), 36 C.P.R.

(4th) 289, [2005] 2 F.C. 654

(F.C.A.) 200, 212, 218, 305

Caron v. Association des Pompiers

(1992), 42 C.P.R. (3d) 292

(F.C.T.D.) 143, 162

CCH Canadian Ltd. v. Law Society

of Upper Canada (2002), 18

C.P.R. (4th) 161 (F.C.A.); varied

CCH Canadian Ltd. v. Law

Society of Upper Canada, [2004]

1 S.C.R. 339, 2004 SCC 13 67,

139, 140, 142, 143, 154, 155,

156, 157, 158, 159, 161, 238,

239, 240, 241, 291, 301

Centre Ice Ltd. v. National Hockey

League (1994), 53 C.P.R. (3d) 34

(F.C.A.) 93

Cheskes et al v. A.G.(Ont.), [2007]

O.J. no. 3515, 2007 CanLII

38387 (Ont. S.C.) 247

Ciba-Geigy Canada Ltd. v. Apotex

Inc, [1992] 3 S.C.R. 120, (1992)

44 C.P.R. (3d) 289 (S.C.C.)

16, 17, 19, 59

Clairol International Corp. v.

Thomas Supply \& Equipment Co.

Ltd. (1968), 55 C.P.R. 176 (Ex.

Ct.) 29, 30, 31, 32

Compagnie Générale des Établissements Michelin-Michelin \& Cie v.

National Automobile, Aerospace,

Transportation and General 
Workers Union of Canada (CAWCanada) (1996), 71 C.P.R. (3d) 348, [1997] 2 F.C. 306, [1996] F.C.J. 1685 (F.C.T.D.) 29, 31, 32, 265, 286

Compo Co. v. Blue Crest Music Inc., [1980] 1 S.C.R. $357 \quad 238$

Compro Communications Inc. $v$. Communications Promo-Phone LT Inc. (1991), 41 C.P.R. (3d) 260 (Que. S.C.) 17

Comstock Canada v. Electec Ltd (1989), 28 C.P.R. (3d) 495

(F.C.T.D.) 278

Consumers Distributing Co. v. Seiko

Time Canada Ltd., [1984] 1

S.C.R. $583 \quad \mathbf{5 , 9 , 5 9}$

Cuisenaire v. Southwest Imports,

[1968] 1 Ex. C.R. 493 274, 290

David v. McArthur (1969), 10

D.L.R. (3d) 250, rev'd (1970) 17

D.L.R. (3d) (B.C.C.A.) $\mathbf{2 5 0}$

Demco Manufacturing Inc. c. Foyer d'artisanat Raymond inc., [2006] J.Q. no 222, 2006 QCCA 5216 Desputeaux v. Éditions Chouette (1987) inc. (2001), 16 C.P.R. (4th) 77 (Que. C.A.); rev'd Desputeaux v. Éditions Chouette (1987) inc., [2003] 1 S.C.R. 178

\section{6, 178, 179, 187}

Director of Investigation and Research v. NutraSweet \& Co. (1990), 32 C.P.R. (3d) 1 (Comp. Trib.) 308

Director of Investigation and

Research v. Warner Music

Canada Ltd. (1997), 78 C.P.R. (3d) 321 (Comp. Trib.) $\mathbf{3 0 8}$

Dolmage v. Erskine (2003), 23

C.P.R. (4th) 495 (O.S.C.J., Small Claims) 188, 190
Dr. Q. v. College of Physicians and Surgeons of British Columbia, [2003] 1 S.C.R. $226 \quad 217$

Dubiner v. MCA Canada Ltd. (1976), 30 C.P.R. (2d) 281 (Ont. H.C.) 29

Dumont Vins \& Spiritueux Inc. v. Celliers du Monde Inc., [1990] R.J.Q. 556 (Que. S.C.) 13

Dumont Vins \& Spiritueux Inc. v. Celliers du Monde Inc., [1992] 2 F.C. 634 (F.C.A.), (1992), 42 C.P.R. (3d) 197 12, 13

Energy Absorption Systems Ltd v. Y. Boissonneault et Fils Inc. (1990), 30 C.P.R. (3d) 420 (F.C.T.D.)

\section{5}

Euro-Excellence Inc. v. Kraft

Canada Inc., 2007 SCC 37, rev'g Kraft Canada Inc. v. Euro Excellence Inc. (2005), 47 C.P.R. (4th) 113 (F.C.A.), rev'g Kraft Canada Inc. v. Euro Excellence Inc. (2003), 25 C.P.R. (4th) 22 (F.C.T.D.) 287, 288, 289, 291, 292, 308

Ferguson v. McBee Technographics Inc. (1989), 24 C.P.R. (3d) 240 (Man. Q.B.) 251

Ferring Inc. v. Canada (Attorney General) (2003), 26 C.P.R. (4th) 155, 2003 FCA $274 \quad \mathbf{9 4 , 9 5}$

First Nations Limited Partnership v. John Doe (3 June 2002), (Ont. S.C.J.) 261

Free World Trust v. Électro Santé Inc., [2000] 2 S.C.R. 1024, 2000 SCC $35 \quad 67$

FWS Joint Sports Claimants v.

Canada (Copyright Board),

[1992] 1 F.C. 487 (F.C.A.) $\mathbf{2 1 3}$ FWS Joint Sports Claimants Inc. v. 
Border Broadcasters Inc. (2001), 16 C.P.R. (4th) 61 (F.C.A.) 215 Garland v. Gemmill (1887), 14

\section{S.C.R. $321 \quad \mathbf{1 2 4}$}

General Motors of Canada Ltd. v.

City National Leasing, [1989] 1

S.C.R. $641 \mathbf{1 0}$

Glaxo Wellcome PLC v. M.N.R.

(1998), 81 C.P.R. (3d) 372

(F.C.A.) 243, 244

Gnass v. Cité d'Alma, (23 November

1973), Roberval A-158 (Que.

C.S.); (30 June 1977), Quebec 09-000032-745 (Que. C.A.)

174

Gould Estate v. Stoddard Publishing

Co. (1996), 31 C.C.L.T. (2d) 224

(Ont. Gen. Div.), rev'd (1998), 39

O.R. (3d) 545 (Ont. C.A.) 248

H.J. Heinz Co. of Canada Ltd. v.

Canada (Attorney General),

[2006] 1 S.C.R. $441 \quad 257,265$,

266

Harvard College v. Canada

(Commissioner of Patents),

[2002] 4 S.C.R. 45, (2002) 219

D.L.R. (4th) 577, 2002 SCC 76

68, 74, 76, 77, 265, 291, 301

Helbronner v. Daoust (1915), 49

C.S. 65, [1917] Droit d'Auteur 58 (Que. S.C.) 167

Hoffmann-La Roche Ltd. v. Canada

(Minister of Health) (2005), 40

C.P.R. (4th) 108, 2005 FCA 140

95

Hollingsworth v. BCTV, a division of

Westcom TV Group Ltd., [1998]

B.C.J. No. 2451, [1999] 6

W.W.R. 54, 59 B.C.L.R. (3d) 121

(B.C.C.A.) 251

Hubert v. Mary (1906), 15 B.R. 381

(Que. C.A.) 113, 122, 136
Imperial Tobacco Co. v. Registrar of Trademarks, [1939] 2 D.L.R. 65

(Ex. Ct.) $\mathbf{2 8 1}$

INAO v. Brick Brewing Co. Ltd.

(1995), 66 C.P.R. (3d) 351

(T.M.O.B.) $\mathbf{6 0}$

INAO v. Pepperidge Farm, Inc.

(1997), 84 C.P.R. (3d) 540

(T.M.O.B.) 61

Institut National des Appellations

d'Origine des Vins et Eaux-de-Vie

['INAO'] et al. v. Andres Wines

Ltd. et al. (1987), 16 C.P.R. (3d)

385 (Ont. H.C.J.), aff'd (1990),

30 C.P.R. (3d) 279 (Ont. C.A.)

60

Insurance Corporation of British

Columbia v. Somosh (1983), 51

B.C.L.R. $344 \mathbf{2 5 0}$

Interlego AG v. Irwin Toy Ltd.

(1985), 3 C.P.R. (3d) 476

(F.C.T.D.) 31, 274

Irwin Toy v. Doe (2000), 12 C.P.C.

(5th) 103 (Ont. S.C.J.) $\mathbf{2 6 1}$

J.M.F. v. Chappell, [1998] B.C.J.

No.276 249

Janssen-Ortho inc. v. Novopharm

Ltd. (2006), 57 C.P.R. (4th) 6,

2006 FC $1234 \quad 93$

John Maryon International Ltd $v$.

New Brunswick Telephone Co.

Ltd (1982), 141 D.L.R. (3d) 193

(N.B.C.A.) 174

Joseph v. Daniels (Brent Daniels

Photography) (1986), 4 B.C.L.R.

(2d) 239, 11 C.P.R. (3d) 544

251

Joubert v. Geracimo (1916), 26 B.R.

97, 35 D.L.R. 683 (Que. K.B.)

113, 122, 166, 167

Kibale v. Canada, [1991] F.C.J. No.

634 (F.C.) (QL) $\mathbf{2 6 1}$ 
Kilvington v. Goldberg (1957), 8 D.L.R. (2d) 768 (Ont. H.C.)

\section{3, 162}

Kirkbi AG v. Ritvik Holdings Inc., [2005] 3 S.C.R. 302, 2005 SCC $653,9,10,11,12,17,18,19$, 21, 24, 59, 269, 279, 281, 282, 290, 291, 304

Lac Minerals Ltd. v. International

Corona Resources Ltd., [1989] 2

S.C.R. 574, [1989] S.C.J. No. 83 253

Langlois v. Vincent (1874), 18

Lower Canada Jurist 160 (Que.

S.C.) 123

Lapierre-Desmarais v. Éditions

Fides, J.E. 99-1424 (C.Q.) 178, 182, 190

Lavigne v. Canada (Office of the

Commissioner of Official

Languages), [2002] 2 S.C.R. 773

265

Les Ateliers Tango Argentin Inc v.

Festival d'Espagne et d'Amérique

Latine Inc. (1997), 84 C.P.R. (3d)

56 (Que. S.C.) 178, 190

Les rôtisseries St-Hubert Ltée v. Le

Syndicat des Travailleur(euse)s

de la rôtisserie St-Hubert de

Drummondville (C.S.N.) (1986),

17 C.P.R. (3d) 461 (Que. S.C.)

9, 16

Loblaw Companies Ltd. v. Aliant

Telecom Inc., [2003] N.B.J. No.

208 (N.B.Q.B.) (QL) 261

MacDonald et al. v. Vapor Canada

Ltd., [1977] 2 S.C.R. 134

$\mathbf{7 , 8}$

Mainetti SpA v. E.R.A. Display Co.

(1984), 80 C.P.R. (2d) 206

(F.C.T.D.) $\mathbf{2 7 8}$

Maple Leaf Broadcasting Company
Limited v. CAPAC, [1954] S.C.R. 624213

Matrox Electronic Systems v.

Gaudreau, [1993] QJ No. 1228

(Que. S.C.) 275

Mattel, Inc. v. 3894207 Canada Inc., [2006] 1 S.C.R. 772 22, 25, 26, 27, 30, 67

McIndoo v. Musson Book Co.

(1916), 35 O.L.R. 342 (Ont.

\section{C.A.) 284}

Medovarski v. Canada (Minister of

Citizenship and Immigration);

Esteban v. Canada (Minister of

Citizenship and Immigration),

[2005] 2 S.C.R. $539 \quad 179$

Merck Frosst Canada Inc. v. Canada

(Minister of National Health and

Welfare) (1994), 55 C.P.R. (3d)

302 (F.C.A.) 93

Milliken \& Co. v. Interface Flooring

(T.D.), [1998] 3 F.C. 103

(F.C.T.D.) 271

Milton v. Savinkoff, [1993] B.C.J.

No. 2396 (B.C.S.C.) $\mathbf{2 5 1}$

Monsanto Canada Inc. v. Schmeiser, [2004] 1 S.C.R. 902, 2004 SCC

$3468,69,70,74,77,78$

Morang and Co. v. LeSueur (1911), 45 S.C.R. 95 165, 178, 299

Morang and Co. v. Publishers' Syndicate (1900), 32 O.R. 393 133

Moreau v. St. Vincent, [1950] Ex. C.R. 198 (Can. Ex. Ct.) 144 Motel 6, Inc. v. No. 6 Motel Ltd. et al. (1981), 56 C.P.R. (2d) 44 (F.C.T.D.) 9

Neighbouring Rights Collective of Canada v. Society of Composers, Authors and Music Publishers of Canada (2003), 26 C.P.R. (4th) 257 (F.C.A.) 214, 216 
New York Herald Co. v. Ottawa

Citizen Co. (1909), 41 S.C.R. 229

284, 285, 290

North West Marine Technology Inc.

v. Crosby (c.o.b. Micro Mark)

(1998), 75 C.P.R. (3d) 491

(B.C.S.C.) 275

Orkin Exterminating Co. Inc. v.

Pestco Co. of Canada Ltd.

(1985), 5 C.P.R. (3d) 433 (Ont.

C.A.) $\mathbf{3 0}$

Oxford Pendaflex Canada Ltd. v.

Korr Marketing Ltd., [1982] 1

S.C.R. $494 \mathbf{5 9}$

Pateman v. Ross, [1988] 68 Man.R.

(2d) $181 \quad 251$

Pioneer Hi-Bred Ltd. v. Canada

(Commissioner of Patents),

[1987] 3 F.C. 8, 77 N.R. 137

(F.C.A); aff'd Pioneer Hi-Bred

Ltd. v. Canada (Commissioner of

Patents), [1989] 1 S.C.R. 1623

68, 72, 73, 74, 76

Pizza Pizza Ltd. v. Canada (1998),

26 C.P.R. (3d) 355 (F.C.A.)

281

Poirier v. Wal-Mart Canada Corp., 2006 BCSC 1138251

Positive Attitude Safety System Inc.

v. Albian Sands Energy Inc.

(2005), 43 C.P.R. (4th) 418

(F.C.A.) $\mathbf{2 4}$

Prise de parole Inc. et al. v. Guérin, éditeur Ltée (1995), 66 C.P.R.

(3d) 257 (F.C.T.D.) 182, 183

Pyrrha Design Inc. v. 623735

Saskatchewan Ltd. (2004), 36

C.P.R. (4th) 432 (F.C.A.) 272

R. v. Boutin, [1997] AQ no. 3111

(Que. C.A.) 276

R. v. Crown Zellerbach Canada Ltd., [1988] 1 S.C.R. 401
R. v. Daoust, [2004] 1 S.C.R. 217 179

R. v. Duarte, [1990] 1 S.C.R. 30

246

R. v. Dyment, [1988] 2 S.C.R. 417

252

R. v. Mac, [2002] 1 S.C.R. $856 \mathbf{1 7 9}$

R. v. O'Connor, [1995] 4 S.C.R. 411 247

R. v. Plant, [1993] 3 S.C.R. 281

246, 248, 254

Re: Anti-Inflation Act, [1976] 2

S.C.R. $373 \quad 249$

Re Application of Abitibi Co. (1982),

62 C.P.R. (2d) 81 (P.A.B) 72, 73

Re Johnston and Frank Johnston's

Restaurants Limited, [1980]

P.E.I.J. No.34 261

Re Private Copying 2003-2004,

Tariff of Levies to be Collected by

CPCC (2003), 28 C.P.R. (4th)

$417 \mathbf{3 0 5}$

Re Royalties for Retransmission

Rights Of Distant Radio and

Television Signals (1990), 32

C.P.R. (3d) 97 (Cop. Bd.) $\mathbf{2 1 3}$

Re SARDEC (1998), 86 C.P.R. (3d)

481 (Cop. Bd.) 206

Re Statement of Royalties to be

Collected by SOCAN and by

NRCC for Pay Audio Services

(Tariff 17.B) (2002), 19 C.P.R.

(4th) 67 (Cop. Bd.) 214

Re Statement of Royalties to be

Collected for Performance in

Canada of Dramatico-musical or

Musical Works in 1990, 1991,

1992 and 1993 (1993), 52 C.P.R.

(3d) 23 (Cop. Bd.); aff'd

Canadian Association of

Broadcasters v. SOCAN (1994),

58 C.P.R. (3d) 190 (F.C.A.) 211 
Reference re Constitution Act, 1867 (1991), 35 C.P.R. (3d) 289 (Man.

\section{C.A.) 13,14}

Remington Rand Corp v. Phillips

Electronics NV (1995), 64 C.P.R.

(3d) 467 (F.C.A.) 280, 281, 283

Remo Imports Ltd. v. Jaguar Cars

Ltd. (2006), 47 C.P.R. (4th) 1

(F.C.T.D.), rev'd on other

grounds, [2008] 2 F.C.R. 132

(F.C.A) 24

Riello Canada, Inc. v. Lambert

(1986), 9 C.P.R. (3d) 324

(F.C.T.D.) 12

Ritchie v. Sawmill Creek Golf \&

Country Club Ltd. (2003), 27

C.P.R. (4th) 220 (O.S.C.J.) varied

Ritchie v. Sawmill Creek Golf \&

Country Club Ltd. (2004), 35

C.P.R. (4th) 163 (O.S.C.J.) 173,

\section{9, 239}

Robertson v. Thomson Corp., [2006]

2 S.C.R. 363239

Rose v. McLean Publishing Co.

(1897), 24 O.A.R. 240 (Ont.

\section{C.A.) 284}

Royal Doulton Tableware Ltd v.

Cassidy's Ltd (1984), [1986] 1

F.C. 357 (F.C.T.D.) 271

Rucker Co. v. Gavel's Vulcanizing

Ltd. (1985), 7 C.P.R. (3d) 294

(F.C.T.D.) 274, 275, 276, 277, 290

Rusche v. Insurance Corp. of British

Columbia, [1992] B.C.J. No. 87,

4 C.P.C. (3d) 12 (B.C.S.C.) 251

$S \& S$ Industries Inc. v. Rowell

(1966), 56 D.L.R. (2d) 501

(S.C.C.) 12

Safematic, Inc. v. Sensodec Oy

(1988), 21 C.P.R. (3d) 12

(F.C.T.D.) 12
Samann v. Canada's Royal Pinetree Mfg Co. Ltd. (1986), 9 C.P.R. (3d) 223 (F.C.A.) $\mathbf{2 8 1}$

Schreiber v. Canada (Attorney

General), [2002] 3 S.C.R. 269

179

Silber v. British Columbia Television

Broadcasting System (1985), 25

D.L.R. (4th) $345 \quad \mathbf{2 5 0}$

Smiles v. Belford (1877), 1 O.A.R. 436 (Ont. C.A.) 122, 133, 136

Smith \& Nephew v. Glen Oak Inc (1996), 68 C.P.R. 3d 153 (F.C.A.) 287

Snow v. The Eaton Centre Ltd. (1982), 70 C.P.R. (2d) 105 (Ont.

H.C.J.) 173, 182, 184, 196

SOCAN - Tariff 22 (Transmission of Musical Works to Subscribers via a Telecommunications Service not covered under Tariff Nos. 16 or

17) (1999), 1 C.P.R. (4th) 417

(Cop. Bd.) 217

Société des Loteries du Québec v.

Club Lotto International CLI Inc.

(2001), 13 C.P.R. (4th) 315

(F.C.T.D.) $\mathbf{2 8 6}$

Society for Reproduction Rights of Authors, Composers and

Publishers in Canadav.

MusiquePlus Inc. (2000), 10

C.P.R. (4th) 242 (Cop. Bd.)

205

Society of Composers, Authors and Music Publishers of Canada v.

Canadian Assn. of Internet

Providers, [2004] 2 S.C.R. 427

201, 217, 234, 235, 236, 237,

240, 266, 305

Society of Composers, Authors and

Music Publishers of Canada v.

Kicks Roadhouse Inc. (c.o.b. 
How-Dee's) (2005), 39 C.P.R. (4th) 238 (F.C.T.D.) 201

Somwar v. McDonald's Restaurant of Canada Ltd. (2006), 79 O.R.

(3d) 172 (Ont. S.C.J.) 249

Source Perrier (Sociéte anonyme) $v$.

Fira-Less Marketing Co. Limited, [1983] 2 F.C. 18 (1983), 70

C.P.R. (2d) 61 (F.C.T.D.) $\mathbf{2 8}$

Spiro-Flex Industries Ltd $v$.

Progressive Sealing Inc. (1986),

32 D.L.R. (4th) 201 (B.C.S.C.)

\section{3}

Sport Maska Inc. v. Canstar Sports

Group Inc., (1994), 57 C.P.R.

(3d) 323 (Que. S.C.) 20

St. Pierre v. Pacific Newspaper

Group Inc., [2006] B.C.J. No.

259 (B.C.S.C.) 251

Statement of Proposed Royalties to

be Collected by CSCS for the

Retransmission of Distant

Television Signals, in Canada, in

2002 and 2003 (14 April 2001),

Copyright Board, online:

$<$ http://www.cb-cda.gc.ca/tariffs/

proposed/r14042000-b.pdf> 215

Statement of Royalties to be

Collected by CBRA for the

Fixation and Reproduction of

Works and Communication

Signals, in Canada, by

Commercial Media Monitors for

the Years 2000-2005 and Non-

commercial Media Monitors for

the Years 2001-2005 (2005) 39

C.P.R. (4th) 152 (Cop. Bd.)

219

Statement of Royalties to be

Collected by ERCC from

Educational Institutions in

Canada, for the Reproduction and Performance of Works or

\section{Other Subject-Matters}

Communicated to the Public by Telecommunication for the Years 2003 to 2006 (14 January 2005), Copyright Board, online: $<$ http://www.cda-cb.gc.ca/decisions/e14012005-b.pdf> 206

Statement of Royalties to be

Collected by SOCAN and NRCC in Respect of Commercial Radio (Tariff 1.A - 2003-2007) (15 October 2005), Copyright Board, online: <http://www.cbcda.gc.ca/tariffs/certified/m15102 005-b.pdf> 214

Statement of Royalties to be Collected by SOCAN and NRCC in Respect of Pay Audio Services (2003-2006) (26 February 2005), Copyright Board, online: <http://www.cb-cda.gc.ca/tariffs/ certified/m26022005-b.pdf>

\section{4}

Statement of Royalties to be

Collected for Performance or

Communication in Canada of

Dramatico-musical Works in

1992, 1993 and 1994 (1994), 58

C.P.R. (3d) 79 (Cop. Bd.) $\mathbf{2 1 3}$

Straka v. Humber River Regional Hospital (2000), 51 O.R. (3d) 1

(Ont. C.A.) 261

Syntox Inc. v. Apotex Inc. (1984), 1

C.P.R. (3d) 145 (F.C.A.) 29

Tat-ha v. Centre hospitalier de

l'Université Laval (1999), BE 99-

259, [1999] AQ 181 (Que. S.C.)

189, 190

Tele-Direct (Publications) Inc. v.

American Business Information

Inc. (1997), 76 C.P.R. (3d) 296

(F.C.A.) 143, 162, 299 
Television Retransmission Tariff 2001-2003 (22 March 2003), Copyright Board, online: <http://www.cb-cda.gc.ca/tariffs/ certified/r22032003-b.pdf> 205

Théberge v. Galerie d'Art du Petit Champlain, [2002] 2 S.C.R. 336, 2002 SCC 34 67, 240, 241, 299

Thomas Betts v. Panduit Corp.(2000), 4 C.P.R. (4th) 498 (F.C.A) $\mathbf{2 8 1}$

Tommy Hilfiger Licensing Inc. $v$. Produits de Qualité I.M.D. Inc., [2005] F.C. 10 (F.C.T.D.) 29

Top Notch Construction Ltd. v. Top

Notch Oilfield Services Ltd. (2001), 13 C.P.R. (4th) 515 (F.C.T.D.) $\mathbf{3 1}$

Toyota Jidosha Kabushiki Kaisha v. Lexus Foods Inc. [2001] 2 F.C. 15, (2000), 9 C.P.R. (4th) 297 (F.C.A.) 26

TV Guide Inc./TV Hebdo Inc. v. Publications La Semaine Inc. et al. (1984), 9 C.P.R. (3d) 368 (Que. S.C.) 19

$U \& R$ Tax v. $H \& R$ Block (1995), 62 C.P.R. (3d) 257 (F.C.T.D.)

\section{1}

United Artists Corp. v. Pink Panther Beauty Corp., [1998] 3 F.C. 534, (1998), 80 C.P.R. (3d) 247 (F.C.A.) 26

Université du Québec à Trois-

Rivières v. Larocque, [1993] 1

S.C.R. $471 \quad 211$

Veuve Clicquot Ponsardin v.

Boutiques Cliquot Ltée, [2006] 1

S.C.R. 824 22, 24, 25, 26, 27,

28, 29, 30, 31, 32, 33, 291, 302

Wa'el Chehab v. John Doe (3

October 2003), (Ont. S.C.J.)

261
Walt Disney Productions v. Triple

Five Corp. (1994), 53 C.P.R. (3d)

129 (Alta. C.A.) 24

WCC Containers Sales Ltd v. Haul-

All Equipment Ltd, [2003] F.C.

962 (F.C.T.D.) 282, 283, 293

Whirlpool Corp. v. Camco Inc.,

[2000] 2 S.C.R. 1067, 2000 SCC

$67 \quad 67$

Wooding v. Little (1982), 24

C.C.L.T. 37, [1982] B.C.J. No. 1422 (B.C.S.C.) (QL) 250

\section{AUSTRALIA}

Meskenas v. ACP Publishing Pty Ltd, [2006] FMCA $1136 \mathbf{1 9 6}$

\section{FRANCE}

Bordeaux, 24 August 1863, S. 1864.II.194 (Delpit et Lacour c. Gué et consorts) 165

Paris, 1ère Ch., 1 February 1989, (1989) 142 R.I.D.A. 301 (Annot. Sirinelli) (Bragance c. Olivier Orban et Michel de Grèce) $\mathbf{1 7 7}$ Trib. civ. Seine, 16 December 1899 ,

D. 1900.II.152 (Agnès dit A. Sorel c. Fayard frères) $\mathbf{1 6 5}$

\section{GERMANY}

Bundesgerichtshof, 27 March 1969 (German Federal Supreme Court), English version available in (1970) 1 I.I.C. 136 (Rote Taube) 71

Bundesgerichtshof, 22 June 1976 (German Federal Supreme Court), 
English version online:

$<$ http://eupat.ffii.org/papers/bghdispo76/index.en.html>

(Dispositionsprogramm)

72

\section{UNITED KINGDOM}

\section{A.G. Spalding \& Bros. v. A. W.}

Gamage (1915), 32 R.P.C. 273, [1914-1915] All E.R. 147 (H.L.)

\section{9}

American Cyanamid v. Ethicon, [1975] A.C. 396 (H.L.) 93 Attorney-General for Ontario $v$. Attorney-General for Canada, [1937] A.C. 405 (P.C.) 4

Bollinger v. Costa Brava Wine Co., [1961] R.P.C. 116, [1961] 1 All E.R. 561 (Ch.D.) 58

British Leyland Motor Corp v.

Armstrong Patents Co., [1986] 1 All E.R. $850 \quad 273$

Confetti Records (a firm) and others v. Warner Music UK Ltd (trading as East West Records), [2003] E.W.H.C. 1274 (Ch.D.)

\section{5}

Cramp v. Smythson, [1944] A.C.

329 143, 162

Erven Warnink B.V. v. J. Townend \&

Sons (Hull) Ltd., [1979] A.C.

731, [1980] R.P.C. 31 (H.L.)

\section{7,58}

Harris v. Davies (1885), 10 App.

Cas. Rep. 282136

Hodge v. The Queen (1883), 9 App.

Cas. Rep. $17 \quad 136$

Hyperion Records Ltd v. Sawkins, [2005] 1 W.L.R. 3281 (England and Wales C.A. (Civil Division)) 188
Interlego A.G. v. Tyco Industries Inc. (1989), 1 A.C. 217275 Jefferys v. Boosey (1854), 24 L. J.

Ex. 81 (H.L.) 127, 128

Kelly v. Morris (1866), L.R. 1 Eq.

69 141, 152

MacMillan \& Co. v. Cooper (1923),

93 L.J.P.C. 113, 40 T.L.R. 186

(J.C.P.C. - India) 143, 162

Manchester Brewery Company,

Limited v. North Cheshire and

Manchester Brewery Company,

Limited, [1898] 1 Ch. $539 \mathbf{5 8}$

Morrison Leahy Music Ltd. v.

Lightbond Ltd., [1993] E.M.L.R.

144 (Ch.D.) 195

Pasterfield v. Denham, [1999]

F.S.R. 168 (Plymouth County

Ct., U.K.) 184, 196

Perry v. Truefitt (1842), 6 Beav. 66,

49 E.R. $749 \quad 17$

Reckitt \& Colman Products Ltd. v.

Borden Inc., [1990] 1 All E.R.

873 (H.L.) 17, 58

Riel v. The Queen (1885), 10 App.

Cas. Rep. 675136

Routledge v. Low (1868), L.R. 3

(H.L.) 100 113, 118, 122, 136

Sayre v. Moore (1785), 1 East 361,

102 E. R. 139 (K.B.) 155

Sawkins v. Hyperion Records Ltd.,

[2004] 4 All E.R. $418 \quad \mathbf{1 8 8 ,} 196$ Seixo v. Provezende (1866), L.R. 1

Ch. App. $192 \mathbf{5 8}$

Singer Manufacturing Co. v. Loog (1880), 18 Ch.D. 395 (C.A.), aff'd (1882), 8 App. Cas. 15 (H.L.) 17

Tidy v. Trustees of the Natural History Museum (1995), 39

I.P.R. 501, [1996] E.I.P.R. D-81

(H.C.) 195, 196 
Universities of Oxford and

Cambridge v. Eyre \&

Spottiswoode Ltd., [1964] $1 \mathrm{Ch}$

736, [1963] 3 All E.R. $289 \mathbf{2 3 8}$

University of London Press $v$.

University Tutorial Press, [1916]

2 Ch. 601 143, 144, 145, 148,

149, 152, 154, 155, 161

Vine Products v. Mackenzie \& Co.

Ltd., [1969] R.P.C. 1 (Ch.D.)

58

Walter v. Lane, [1900] A.C. 539

143, 151, 152, 160, 161

\section{UNITED STATES OF AMERICA}

Carter v. Helmsley-Spear, 852

F.Supp 228 (S.D.N.Y. 1994); 861

F.Supp 303 (S.D.N.Y. 1994); 71

F.3d 77 (2nd Cir. 1995) 184,

196

Clemens v. Belford, Clarke \& Co.

(1883), 14 F. 728 (N.D. Ill.)

284

Diamond, Commissioner of Patents and Trademark v. Chakrabarty

(1980), 447 U.S. 303, 206

U.S.P.Q. $193 \quad \mathbf{6 8 , 7 0 , 7 2 , ~ 7 5 , ~} 76$

Ex Parte Allen, 2 U.S.P.Q.2d 1425

(B.P.A.I. 1987) $\mathbf{7 2}$

Feist Publications, Inc. v. Rural

Telephone Service Co., 499 U.S.

340 (1991) 142, 157, 299

Flack v. Friends of Queen

Catherine Inc et al., 139 F.Supp

2d 526 (S.D.N.Y. 2001) 196

International News Service v.

Associated Press, 248 U.S. 215

(1918) 33, 150

Martin v. City of Indianapolis, 982
F.Supp 625 (S.D. Ind. 1997), 4 F.Supp 2d 808 (S.D. Ind. 1998), 28 F.Supp 2d 1098 (S.D. Ind. 1998); 192 F.3d 608 (7th Cir. 1999) 196

Metro-Goldwyn-Mayer Studios Inc. et al. v. Grokster, Ltd. et al., 545 U.S. 913 (2005) 228

Moseley v. V Secret Catalogue Inc., 537 U.S. 418 (2003) 28, 33

Phillips v. Pembroke Real Estate,

Inc., 288 F.Supp 2d 89 (D. Mass. 2003) 196

Pillsbury-Washburn Flour Mills Co. v. Eagle, 86 F. 608 (7th Cir. 1898) 59

Pollara v. Seymour and Casey, 150 F.Supp 2d 393 (S.D.N.Y. 2001); 206 F.Supp 2d 333 (N.D.N.Y. 2002) 196

Sheldon v. Metro-Goldwyn Pictures Corp., 81 F.2d 49 (2d Cir. 1936) 145

State of Florida, Dept. of Citrus v. Real Juices, Inc, 330 F. Supp. 428 (M.D. Fla. 1971) 54

Tasini et al. v. New York Times et al., 121 S. Ct. 2381 (2001)

221

\section{EUROPEAN UNION}

The President and Fellows of Harvard College v. British Union for the Abolition of Vivisection et al. (6 July 2004), Boards of Appeal of the European Patent Office Decision T-0315/03, online: <http://legal.europeanpatent-office.org/dg3/pdf/ t030315ex1.pdf> $\mathbf{7 1}$ 
WORLD TRADE

ORGANIZATION (WTO)

Canada - Patent Protection of Pharmaceutical Products (Complaint by the European Communities and their Member States) (2000), WTO Doc.
WT/DS114/R (Panel Report) 91

Canada - Term of Patent Protection (2000), WTO Doc. WT/DS170/R (Panel Report) 97

Canada - Term of Patent Protection (2000), WTO Doc.

WT/DS170/AB/R (Appellate

Body Report) $\mathbf{9 8}$ 
Ysolde Gendreau - 9781848445024

Downloaded from PubFactory at 04/26/2023 02: 09 :39PM

via free access 